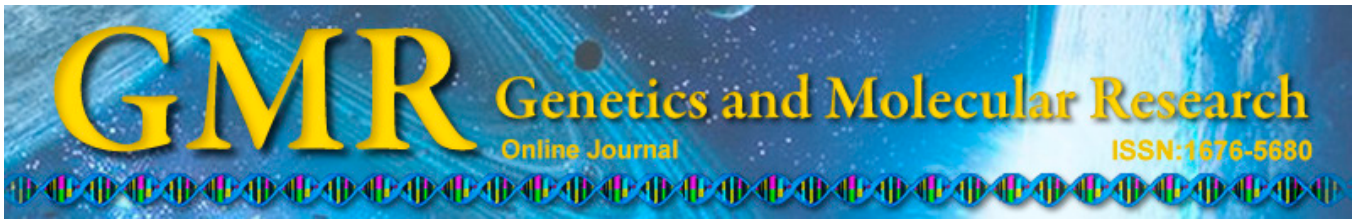

\title{
microRNAs in avian influenza virus H9N2-infected and non-infected chicken embryo fibroblasts
}

\author{
X. Peng, Q.S. Gao, L. Zhou, Z.H. Chen, S. Lu, H.J. Huang, C.Y. Zhan \\ and M. Xiang \\ Animal Biotechnology Laboratory, \\ Wuhan Institute of Animal and Veterinary Science, \\ Wuhan Academy of Agricultural Science \& Technology, Wuhan, China \\ Corresponding author: X. Peng \\ E-mail: pengxiahao@163.com \\ Genet. Mol. Res. 14 (3): 9081-9091 (2015) \\ Received November 3, 2014 \\ Accepted April 29, 2015 \\ Published August 7, 2015 \\ DOI http://dx.doi.org/10.4238/2015.August.7.17
}

\begin{abstract}
There is limited information about microRNAs (miRNAs) in H9N2 subtype influenza virus-infected chicken cells or tissues. In this study, 10,487,469 and 13,119,795 reads were obtained from infected and non-infected chicken embryo fibroblasts, respectively. Seven hundred and thirty-six and 1004 miRNAs, including mature miRNAs and precursors, were obtained from the infected and non-infected fibroblasts, respectively. Of those miRNAs, 48 were expressed differently between the groups: 37 had a low expression level in the infected chicken embryo fibroblast, and the remaining 11 had a higher expression level. Every miRNA was predicted to target immune response-related genes. It has been found that some of the miRNAs, such as gga-miR146c, gga-miR-181a, gga-miR-181b, gga-miR-30b, gga-miR-30c, ggamiR-30e, and gga-miR-455, are expressed differently in other types of influenza-infected chicken cells or tissues.
\end{abstract}

Key words: Avian influenza virus; Chicken embryo fibroblast; H9N2; miRNA 


\section{INTRODUCTION}

Avian influenza viruses (AIV) are highly variable viruses (Guan et al., 1999) that can spread across species (Wan et al., 2008). H9N2, the most common influenza virus in Asia, is an AIV that can infect humans (Guo et al., 1999; Peiris et al., 1999), mice, and swine, as well as birds. In some animals, it can cause moderate disease. Infection in chickens typically leads to moderate respiratory symptoms and a serious drop in egg production (Naeem et al., 2003). As a result of its prevalence and its impact on chickens, there have been large economic losses in poultry production, especially in chicken production. Thus, serious efforts have been made to develop new intervention strategies for controlling H9N2 infection and circulation. Various subtypes of AIV, such as H5N1 (Cong et al., 2007) and H7N9 (Liu et al., 2013), can become highly pathogenic. As a result, more effective strategies are required to control AIV.

microRNAs (miRNAs) are 19-25 nucleotide, non-coding RNAs that regulate the expression of their target messenger RNAs (mRNAs). Thousands of miRNAs have been mapped in the genomes of different organisms, including animals, plants, and microorganisms. Currently, 996 mature miRNAs and 734 precursors have been mapped in the chicken genome. They have roles in almost every life process such as growth, development, breeding, immune response, etc. (Burnside and Morgan, 2011; Powder et al., 2012; Meunier et al., 2013).

Past research has identified several miRNAs as important to a host's immune response against AIV infection. Wang et al. $(2009,2012)$ determined that the expression levels of many miRNAs are influenced by AIV infection; many miRNAs, such as gga-mir-146 and gga-mir-155, target immune-related genes. Additionally, Terrier et al. (2013) found that gga-mir-146a is an important gene in H1N1 and H3N2 influenza virus infections. There are great differences in the expression of miRNAs in H5N3, H1N1, and H3N2. The functions and pathways of miRNAs in different types of AIV may differ. In addition to the miRNAs already mentioned, other miRNAs, such as gga-mir-26a, which is responsible for regulating interleukin 2 in chicken lymphoma lines (Clark et al., 2011), have been identified as regulators of immune-related genes.

Additional information can be obtained from these miRNAs through high-throughput miRNA sequencing, which has been utilized in miRNA research for many years. More specifically, this sequencing method is a good means of acquiring the sequence and expression information of selected functional miRNAs and their target genes.

Chicken embryo fibroblasts can be used to observe cytopathic effects and investigate immune-related gene expression when infected with the influenza virus; they are good model cells for influenza virus culture and research, and have been used for more than 50 years (Scholtissek and Rott, 1969). They can also be used as a good model for studying immune responses in vitro (Barber et al., 2013).

In this study, we used sequencing-by-synthesis with a paired-end $100 \mathrm{bp}$ model in the HiSeq 2000 sequencer (Illumina, San Diego, CA, USA) to study the sequence information and expression levels of miRNAs in both AIV H9N2-infected and non-infected chicken embryo fibroblasts. The purpose of our research was to determine the miRNAs that are important for an immune response to AIV infection. 


\section{MATERIAL AND METHODS}

\section{Virus}

The H9N2 avian influenza virus (Zhang et al., 2011) was propagated, and then a titer was conducted on a 9-day-old specific pathogen-free chicken embryo. The allantoic fluid was obtained 2 days after infection. The fluid was filtered using a $6-\mu \mathrm{m}$ filter after freezing and thawing twice, and then stored at $-80^{\circ} \mathrm{C}$ until required.

\section{Fibroblast, virus infection, and cell collection}

Three 7-day-old specific pathogen-free chicken embryos were euthanized. The fibroblasts were divided using a previously described method (Schat and Purchase, 1998). Next, the fibroblasts were cultured in high glucose Dulbecco's modified Eagle's medium with $10 \%$ calf serum, and $24 \mathrm{~h}$ after division, the cells were passaged at a ratio of 1:4. The cells were then divided into two groups: one group was treated with a $100-\mu \mathrm{L}$ solution of H9N2-infected allantoic fluid diluted with phosphate-buffered saline; the other group was treated with an equal volume of phosphatebuffered saline. When the virus-treated cells exhibited cytopathic effects, and $10 \%$ of them had been shed, the culture was collected to detect the virus titers using a blood agglutination test. If the titer of the virus test was higher than $2^{5}$, the cells were eluted with TRIzol (Invitrogen, Carlsbad, CA, USA), frozen immediately after isolation, and stored in liquid nitrogen for RNA isolation.

\section{RNA isolation}

The total RNA was isolated from the freshly frozen fibroblasts using TRIzol (Invitrogen), according to the manufacturer protocol. Following the RNA isolation, the DNA was digested using DNase I, according to the manufacturer protocol. The RNA samples of the same group were mixed according to the RNA number. The quality of the isolated RNA was assessed on a $1 \%$ agarose gel based on the relative abundance of the $18 \mathrm{~S}$ and $28 \mathrm{~S}$ subunits of ribosomal RNA.

\section{RNA sequencing and bioinformatic analysis}

RNAs were linked with T4 RNA ligase 2, reverse transcribed, and then purified by polyacrylamide gel electrophoresis. After enriching for molecules in the 17-35 nucleotide range by synthesis, the sequencing was carried out with a paired-end $100 \mathrm{bp}$ model in a HiSeq 2000 sequencer (Illumina) at Sangon Biotech Co., Ltd.'s high throughput sequencing center.

The reads obtained from the sequence were analyzed by cutadapt-1.2.1 and quality score pretreatment, which included extracting adapter sequences and low-quality sequences.

\section{Sequence annotation and known miRNA certification}

The sequences of the chicken genome, non-coding RNAs, and mRNAs were downloaded from the ensemble database (http://www.ensembl.org/Gallus_gallus/Info/Index). The acquired reads were compared to the downloaded reads; those that were mismatched by only one nucleotide or those that matched perfectly were deleted. The rest of the reads were matched to the mature miRNAs in the miRBase database that allowed for one nucleotide mismatch. (http://www.mirbase.org/cgi-bin/mirna_summary.pl?org=gga). 


\section{Known miRNA analysis}

The value of the reads per million (RPM) was analyzed as follows:

$$
\mathrm{RPM}=\frac{\text { mappedreads }^{*} 1000000}{\text { totalreads }}
$$

\section{Novel miRNA prediction}

Other sequences for mRNAs, transfer RNAs, ribosomal RNAs, small nuclear RNAs, small nucleolar RNAs, and miscellaneous RNAs were wiped off by comparing to the chicken's genome, and then novel miRNAs were predicted using the miRDeep2 algorithm. The results that were previously unknown were considered to be novel miRNAs.

\section{Expression differences of miRNAs}

The comparison of the expression levels of miRNAs was carried out by detecting different genes based on sequencing according to the method described by Audic and Claverie (1997). For screening the different genes, $P \geq 0.05$, and $\mid \log 2$ Fold changes $\mid \geq 1$.

\section{Enrichment analysis of target genes}

The enrichment analysis of target genes was carried out by gene ontology (GO) and pathway enrichment analysis to determine the target genes and the possible roles of differently expressed miRNAs

\section{RESULTS}

\section{Sequence analysis of small RNAs in chicken embryo fibroblast}

To study the miRNAs involved in a chicken embryo fibroblast affected by influenza A virus H9N2 infection, two small RNA libraries were generated from the infected and noninfected chicken embryo fibroblasts. The libraries were sequenced by the Illumina small RNA deep sequencing platform. In total, 10,487,469 and 13,119,795 raw reads were obtained from the infected and non-infected fibroblasts, respectively. After filtering the low-quality sequences, adapter sequences, sequences with polyA, and sequences that were too long or too short, $4,652,861$ and 8,387,816 clean reads of 18-35 nucleotides were selected for further analysis from the infected and non-infected fibroblasts, respectively.

The annotation of these clean reads revealed that 14,763 and 15,322 clean reads were attributable to miRNA in the infected and non-infected fibroblasts, respectively, whereas the remaining were coding regions, ribosomal RNAs, small nucleolar RNAs, transfer RNAs, miscellaneous RNAs, or small nuclear RNAs. Their distribution is illustrated in Table 1, Figure 1, and Figure 2.

The size distribution of the small RNAs (primarily from 21 to 23 nucleotides) was similar in the two libraries (as shown in Figure 3).

The comparison results of the most abundant miRNA in the two libraries are shown in Table 2. 
Table 1. Sequences, annotation, and known microRNAs (miRNAs) certification.

\begin{tabular}{|c|c|c|c|c|c|c|c|c|c|}
\hline Sample & & All & cds & rRNA & snoRNA & tRNA & miscRNA & snRNA & miRNA \\
\hline \multirow{4}{*}{$\begin{array}{l}\text { QInfected } \\
\text { group } \\
\text { Non-infected group }\end{array}$} & Unique & 327,630 & $62,541.33$ & $13,278.33$ & $3,719.833$ & 642.5 & 2302.5 & 997.5 & 736 \\
\hline & All & $4,652,861$ & $234,919.3$ & $341,900.3$ & $15,323.33$ & 1911.5 & $19,545.5$ & 6,828 & 14,763 \\
\hline & Unique & 502,773 & $90,676.5$ & 18,283 & $7,026.5$ & 1060 & 4331.5 & $2,287.5$ & 1,004 \\
\hline & All & $8,387,816$ & 329,241 & $391,857.5$ & 32,354 & 4011 & 30,739 & $20,172.5$ & 15,322 \\
\hline
\end{tabular}

cds = coding regions; $r$ RNA = ribosomal RNA; snoRNA = small nucleolar RNA; tRNA = transfer RNA; miscRNA $=$ miscellaneous RNA; snRNA = small nuclear RNA.

\section{Uniq SRNA(case1)}

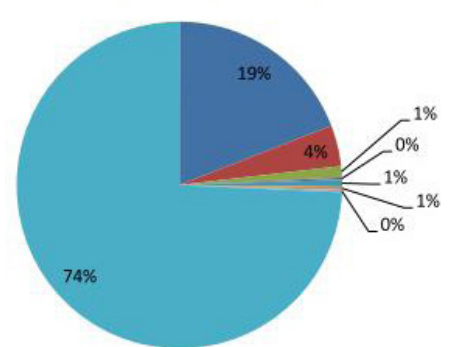

All sRNA(case1)

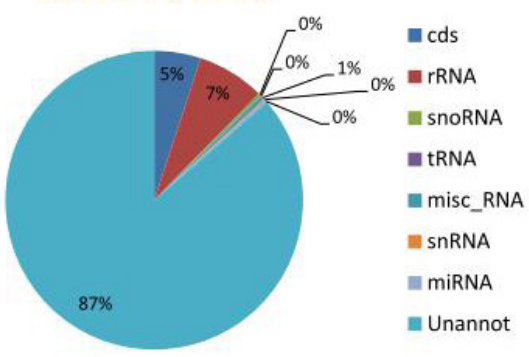

Figure 1. Annotation of sRNAs in the infected group.

\section{Uniq sRNA(control1)}

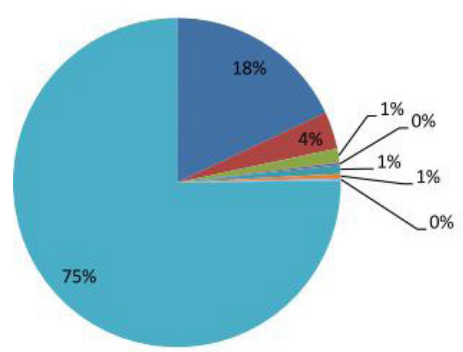

\section{All sRNA(control1)}

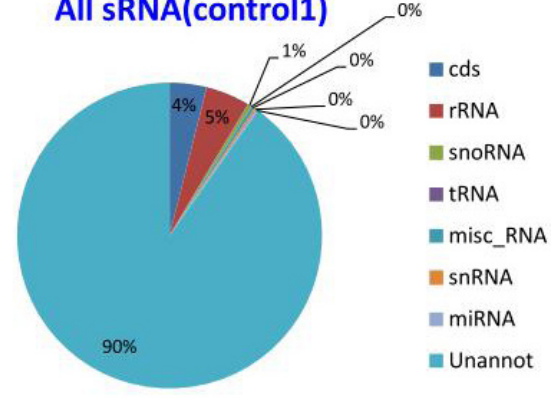

Figure 2. Annotation of sRNAs in the non-infected group.

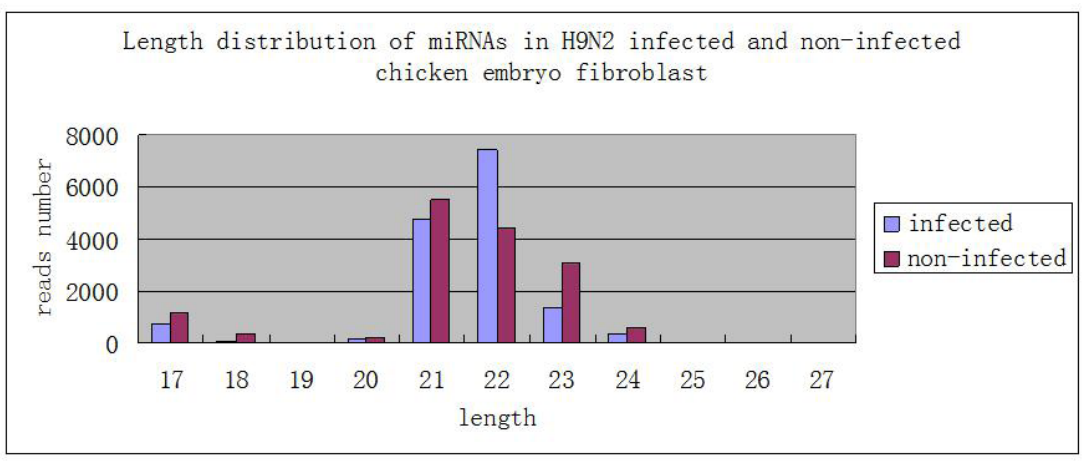

Figure 3. Length distribution of miRNAs in H9N2 infected and non-infected chicken embryo fibroblast. 
Table 2. Ten most abundantly expressed microRNAs (miRNAs).

\begin{tabular}{llrr}
\hline miRNA & Precursor & Infected & Non-infected \\
\hline gga-miR-92-3p & gga-mir-92 & $226,098.15$ & $225,681.19$ \\
gga-miR-181a-5p & gga-mir-181a-1 & $49,446.26$ & $176,864.08$ \\
gga-miR-10b-5p & gga-mir-10b & $188,336.10$ & $26,921.19$ \\
gga-miR-10a-5p & gga-mir-10a & $133,809.73$ & $32,990.70$ \\
gga-miR-214 & gga-mir-214 & $18,288.35$ & $75,248.82$ \\
gga-miR-2954 & gga-mir-2954 & $48,768.92$ & $25,256.98$ \\
gga-miR-222a & gga-mir-222a & $20,388.12$ & $32,566.49$ \\
gga-miR-100-5p & gga-mir-100 & $35,255.87$ & $16,805.35$ \\
gga-miR-125b-5p & gga-mir-125b & $14,698.41$ & $30,673.85$ \\
gga-miR-146c-5p & gga-mir-146c & $32,681.95$ & $8,614.78$ \\
\hline
\end{tabular}

\section{Identification of known miRNAs in the chicken embryo fibroblast}

To investigate the expression of known miRNAs in the chicken embryo fibroblasts, the sequencing results were compared with known mature miRNAs and precursors in miRBase (version 14, 2009 and version 19, 2012). The results showed that 168 mature miRNAs from 145 precursors were found in the two libraries. Among these mature miRNAs, 21 were found exclusively in the infected chicken embryo fibroblast, and 50 were found exclusively in the non-infected chicken embryo fibroblast. Ninety-seven known miRNAs were expressed in both groups.

The following ten miRNAs were dominantly expressed in the two libraries shown in Table 2. For the infected fibroblast, the most abundant miRNA was gga-miR-92-3p, which had 226,098 reads, followed by gga-miR-10b-5p and gga-miR-181a-5p, which had 133,809 and 49,446 reads, respectively. However, in the non-infected group, the most abundant miRNAs were gga-miR-92-3p, gga-miR-181a-5p, and gga-miR-10b-5p, which had 225,681, 176,864, and 32,990 reads, respectively. The RPMs of the miRNAs are shown in Table S1.

\section{Prediction of novel miRNAs}

Forty-four novel miRNAs have been mapped in the chicken genome. From among these miRNAs, 22 were found in both groups, nine were found only in the infected fibroblast, and 13 were found only in the non-infected group. Fourteen miRNAs had reads greater than 500 , all of which were expressed in both groups. The descriptions of the prediction of novel miRNAs are shown in Table $\mathbf{S 2}$.

\section{Differently expressed miRNAs in infected and non-infected fibroblasts}

To survey the differences in miRNA expression, the expression information associated with the infected and non-infected groups was compared.

There were 48 miRNAs that significantly differed in expression between the case and control groups (Table 3). Of these miRNAs, 11 were upregulated in the infected group, whereas the others had lower expression levels in the non-infected group. 


\begin{tabular}{|c|c|c|c|c|c|c|}
\hline miRNA & Infected RPM & Non-infected RPM & $\log 2$ (fold-change) & $P$ value & False discovery rate (FDR) & Type \\
\hline gga-let-7c-5p & $1,117.62$ & $2,480.01$ & -1.1499 & 0.0041 & 0.0205 & Down \\
\hline gga-let-7i & 338.67 & $1,240.01$ & -1.8724 & 0.0054 & 0.0245 & Down \\
\hline gga-miR-106-3p & 0 & 522.11 & NA & 0.0046 & 0.0218 & Down \\
\hline gga-miR-125b-3p & $1,354.69$ & $3,002.12$ & -1.148 & 0.0022 & 0.0114 & Down \\
\hline gga-miR-125b-5p & $14,698.41$ & $30,673.85$ & -1.0613 & $1.36 \mathrm{E}-12$ & $1.67 \mathrm{E}-11$ & Down \\
\hline gga-miR-126-5p & 0 & 391.58 & NA & 0.01778 & 0.0739 & Down \\
\hline gga-miR-128-3p & $1,083.76$ & $2,806.33$ & -1.3726 & 0.0007 & 0.0039 & Down \\
\hline gga-miR-130b-5p & 406.41 & $2,741.07$ & -2.7537 & $1.14 \mathrm{E}-07$ & $9.84 \mathrm{E}-07$ & Down \\
\hline gga-miR-133a-3p & 67.74 & $1,555.44$ & -4.5218 & 2.37E-06 & $1.83 \mathrm{E}-05$ & Down \\
\hline gga-miR-140-3p & $3,522.20$ & $17,882.2$ & -2.34398 & 0 & 0 & Down \\
\hline gga-miR-1456-5p & 406.41 & $1,696.85$ & -2.0619 & 0.0005 & 0.0029 & Down \\
\hline gga-miR-155 & 135.47 & $1,305.27$ & -3.2684 & $9.39 \mathrm{E}-05$ & 0.0006 & Down \\
\hline gga-miR-1552-3p & 0 & 391.58 & NA & 0.0178 & 0.073939 & Down \\
\hline gga-miR-15b-5p & 0 & 750.53 & NA & 0.0006 & 0.0036 & Down \\
\hline gga-miR-15c-5p & 67.73 & $4,927.39$ & -6.1849 & 0 & 0 & Down \\
\hline gga-miR-181a-5p & $49,446.26$ & $176,864.08$ & -1.8388 & 0 & 0 & Down \\
\hline gga-miR-181b-5p & $6,434.78$ & $31,979.11$ & -2.3132 & 0 & 0 & Down \\
\hline gga-miR-184-3p & 474.14 & $1,892.64$ & -1.997 & 0.0003 & 0.0019 & Down \\
\hline gga-miR-199-3p & $3,251.26$ & $10,637.95$ & -1.7101 & $5.68 \mathrm{E}-14$ & $7.91 \mathrm{E}-13$ & Down \\
\hline gga-miR-199-5p & 541.88 & $3,850.54$ & -2.829 & $1.50 \mathrm{E}-10$ & $1.49 \mathrm{E}-09$ & Down \\
\hline gga-miR-205b & 135.47 & $3,654.76$ & -4.7537 & $1.51 \mathrm{E}-14$ & $2.42 \mathrm{E}-13$ & Down \\
\hline gga-miR-214 & $18,288.35$ & $75,248.82$ & -2.0407 & $1.03 \mathrm{E}-12$ & $1.33 \mathrm{E}-11$ & Down \\
\hline gga-miR-221-3p & $1,354.69$ & $3,132.65$ & -1.2094 & 0.0011 & 0.0062 & Down \\
\hline gga-miR-26a-5p & $4,673.69$ & $9,463.21$ & -1.01777 & $6.77 \mathrm{E}-07$ & $5.41 \mathrm{E}-06$ & Down \\
\hline gga-miR-30b-5p & 0 & 326.32 & NA & 0.0349 & 0.1344 & Down \\
\hline gga-miR-30c-5p & $1,354.70$ & $4,633.7$ & -1.7742 & $1.21 \mathrm{E}-07$ & $1.00 \mathrm{E}-06$ & Down \\
\hline gga-miR-30d & $4,199.55$ & $20,101.16$ & -2.259 & 0 & 0 & Down \\
\hline gga-miR-30e-3p & 67.73 & 522.11 & -2.9465 & 0.0249 & 0.0998 & Down \\
\hline gga-miR-30e-5p & 541.88 & $1,370.53$ & -1.3387 & 0.0212 & 0.0863 & Down \\
\hline gga-miR-455-5p & 541.88 & $1,631.59$ & -1.5902 & 0.0041 & 0.0205 & Down \\
\hline gga-miR-460a-5p & 406.41 & $2,023.17$ & -2.3156 & $3.95 \mathrm{E}-05$ & 0.0003 & Down \\
\hline gga-novel-2-mature & 0 & 391.58 & NA & 0.01778 & 0.0739 & Down \\
\hline gga-novel-2-star & 67.73 & 587.37 & -3.1164 & 0.0138 & 0.0614 & Down \\
\hline gga-novel-58-mature & $2,844.85$ & $15,728.5$ & -2.467 & $5.71 \mathrm{E}-14$ & 7.91E-13 & Down \\
\hline gga-novel-59-mature & 67.73 & $1,174.74$ & -4.1164 & $5.59 \mathrm{E}-05$ & 0.0004 & Down \\
\hline gga-novel-69-mature & $1,286.96$ & $2,741.07$ & -1.0908 & 0.005 & 0.0231 & Down \\
\hline gga-novel-79-mature & 67.73 & $1,174.74$ & -4.1164 & $5.59 \mathrm{E}-05$ & 0.0004 & Down \\
\hline gga-let-7f-5p & $3,894.74$ & $1,272.64$ & 1.6137 & $4.60 \mathrm{E}-06$ & $3.42 \mathrm{E}-05$ & $\mathrm{Up}$ \\
\hline gga-miR-100-5p & $35,255.87$ & $16,805.35$ & 1.0689 & $1.07 \mathrm{E}-23$ & $1.85 \mathrm{E}-22$ & Up \\
\hline gga-miR-10a-5p & $133,809.73$ & $32,990.7$ & 2.0201 & $1.75 \mathrm{E}-216$ & $4.55 \mathrm{E}-215$ & $\mathrm{Up}$ \\
\hline gga-miR-10b-5p & $188,336.10$ & $26,921.19$ & 2.8065 & 0 & 0 & Up \\
\hline gga-miR-146c-5p & $32,681.95$ & $8,614.78$ & 1.9236 & $8.12 \mathrm{E}-51$ & $1.69 \mathrm{E}-49$ & Up \\
\hline gga-miR-215-5p & $2,573.92$ & 65.26 & 5.3016 & $3.65 \mathrm{E}-11$ & 4.22E-10 & Up \\
\hline gga-miR-99a-5p & $2,540.05$ & $1,142.11$ & 1.1532 & 0.0042 & 0.0205 & Up \\
\hline gga-novel-1-mature & $57,235.75$ & $17,229.56$ & 1.732 & $2.70 \mathrm{E}-76$ & $6.24 \mathrm{E}-75$ & Up \\
\hline gga-novel-82-mature & 338.67 & 0 & NA & 0.0279 & 0.1096 & Up \\
\hline gga-novel-85-mature & $2,370.72$ & 65.26 & 5.183 & $2.86 \mathrm{E}-10$ & $2.59 \mathrm{E}-09$ & Up \\
\hline gga-novel-94-mature & $2,370.72$ & 65.26 & 5.183 & $2.86 \mathrm{E}-10$ & $2.59 \mathrm{E}-09$ & Up \\
\hline
\end{tabular}

\section{Target prediction and function annotation}

The miRNA prediction results showed that each miRNA might target hundreds of genes. In fact, every miRNA might target immune response-related genes. The target genes of differently expressed miRNAs are shown in Table $\mathbf{S 3}$.

The GO annotation enrichment results showed that the regulation of biological processes, cellular metabolic processes, and developmental processes were the most significantly enriched GO terms. The pathway analysis showed 17 pathways that were enriched with 
miRNA targets (Table 4). The top ten pathways were: ribosome biogenesis in eukaryotes, RNA transport, arginine and proline metabolism, ubiquitin-mediated proteolysis, protein processing in the endoplasmic reticulum, pyrimidine metabolism, basal transcription factors, ovarian steroidogenesis, the NF-kappa B signaling pathway, and SNARE interactions in vesicular transport.

\section{Table 4. Enriched pathways of target genes for differentially expressed microRNAs (miRNAs).}

\begin{tabular}{|c|c|c|c|c|c|}
\hline Pathway ID & All gene number & DEGs number & $P$ value & FDR & Name \\
\hline ko03008 & 68 & 49 & 0.0016 & 0.277 & Ribosome biogenesis in eukaryotes \\
\hline ko03013 & 136 & 90 & 0.0022 & 0.277 & RNA transport \\
\hline ko00330 & 47 & 35 & 0.003 & 0.277 & Arginine and proline metabolism \\
\hline ko04120 & 128 & 84 & 0.0044 & 0.277 & Ubiquitin-mediated proteolysis \\
\hline ko04141 & 150 & 97 & 0.0044 & 0.277 & Protein processing in endoplasmic reticulum \\
\hline ko00240 & 92 & 62 & 0.0056 & 0.2872 & Pyrimidine metabolism \\
\hline ko03022 & 39 & 29 & 0.007 & 0.2872 & Basal transcription factors \\
\hline ko04913 & 47 & 34 & 0.0074 & 0.2872 & Ovarian steroidogenesis \\
\hline ko04064 & 85 & 57 & 0.009 & 0.313 & NF-kappa B signaling pathway \\
\hline ko04130 & 31 & 23 & 0.0167 & 0.5197 & SNARE interactions in vesicular transport \\
\hline ko05014 & 44 & 31 & 0.0185 & 0.5259 & Amyotrophic lateral sclerosis \\
\hline ko03030 & 30 & 22 & 0.0235 & 0.6102 & DNA replication \\
\hline ko00600 & 50 & 34 & 0.0299 & 0.673 & Sphingolipid metabolism \\
\hline ko00010 & 55 & 37 & 0.0302 & 0.673 & Glycolysis/gluconeogenesis \\
\hline ko03450 & 12 & 10 & 0.0357 & 0.7429 & Non-homologous end-joining \\
\hline ko00071 & 36 & 25 & 0.0423 & 0.7582 & Fatty acid degradation \\
\hline ko00460 & 5 & 5 & 0.0455 & 0.7582 & Cyanoamino acid metabolism \\
\hline
\end{tabular}

$\mathrm{DEGs}=$ differentially expressed genes; FDR $=$ false discovery rate.

\section{DISCUSSION}

The Illumina deep sequencing platform is effectively used in discovering miRNAs and widely used for generating small RNA profiles in various organisms and cells (Langmead and Salzberg, 2012).

Thousands of miRNAs have been identified as playing important roles in gene expression, while the exact role in influenza virus infection is still under investigation. Several studies have been carried out on high-pathogenicity influenza viruses, such as H5N1, H1N1, and H5N9.

In the present study, we obtained detailed miRNA profiles of both an influenza A virus H9N2-infected chicken embryo fibroblast and a non-infected chicken embryo fibroblast. To our knowledge, this is the first report that details the miRNA expression profiles of chicken cells infected with AIV H9N2. An inspection of the miRNAs revealed that the majority of the miRNAs that are similar to those expressed in this study are also found in H5N3-, H7N7-, or H1N1-infected chicken lungs or tracheas, although some are different.

The ten most abundant miRNAs present in the chicken fibroblast were as follows: ggamiR-92, gga-miR-181a, gga-miR-10b, gga-miR-10a, gga-novel-68-mature, gga-miR-214, gga-novel-1-mature, gga-miR-2954, gga-miR-222a, and gga-miR-100. gga-miR-92 has been found in avian macrophage cell lines HD11 and IAH30 (Yao et al., 2013). In addition, ggamiR-181a-5p is important for the immune response to either AIV or Marek's disease virus infection (Yao et al., 2009; Lian et al., 2012). Overall, it is considered to be a critical component of the immune system, particularly as it was identified to be an intrinsic modulator of T cell sensitivity and selection in T cell maturation and antigen recognition (Li et al., 2007).

In other species, such as humans, many miRNAs have been identified as important factors in the immune response to AIV infection. For example, mir-15b has been found to 
regulate IP-10/CXCL-10 expression in H9N2-infected human umbilical vein endothelial cells (Chan et al., 2011), and gga-mir-10b and gga-mir-10a are expressed in developing chicken immune organs (Hicks et al., 2009). However, many miRNAs that are important in other influenza viruses do not differ, for example, mir-141, mir-21*, mir-100*, etc. (Lam et al, 2013).

The result of the differential expression analysis indicated that 48 miRNAs were differently expressed in the infected and non-infected chicken embryo fibroblasts. Eleven of those were upregulated and the rest were downregulated in the infected fibroblast. Several of those miRNAs are differentially expressed in other types of influenza virus, as well as in other kinds of viral infections. For example, the expression of gga-mir-146c and gga-mir-155 has been found to influence H5N3 influenza virus infection (Wang et al., 2009, 2012). Additionally, gga-mir-15b, -15c, -181a, -181b, -1456, -140, -26a, -30b, -30c, -30d, -30e, -455-5p, and -99a have been found to influence H5N9 AIV infection in chicken lungs and tracheas. Many miRNAs have been found in viral infections other than influenza. For example, gga-mir-26a, $-30 \mathrm{c},-30 \mathrm{~d},-181 \mathrm{a},-181 \mathrm{~b},-221$, and gga-let-7i are differently expressed in a normal T cell line and a Marek's disease-transformed T-cell line (Yao et al., 2008).

The target gene prediction of the differentially expressed miRNAs, including ggamir-146c, gga-mir-155, gga-mir-26a, gga-mir-30c, gga-mir-30d, gga-mir-181a, gga-mir-181b, gga-mir-221, gga-mir-125b, gga-mir-1456, etc., revealed that they may all target immunerelated genes. Some miRNAs, such as gga-mir-30d, gga-mir-155, and gga-mir-140, are important in the immune response to AIV infection. However, others, such as gga-mir-26a, ggamir-15b, and gga-mir-10b, are important for a host's immune response to other viruses, such as Marek's disease virus, avian leukosis virus, and reticuloendotheliosis virus.

Most of the functions of these miRNAs and the mechanisms by which they act are still unknown, although gga-mir-146c has been identified as an inhibitor of the signaling proteins of the innate immune response controlled by NF-kappa B. Furthermore, gga-mir-26a in transformed avian lymphocyte lines regulates the expression of interleukin-2, which is an important factor in the development, differentiation, proliferation, and homeostasis of $\mathrm{T}$ cells (Xu et al., 2010). Some other miRNAs, such as gga-let-7i, gga-mir-125b, gga-mir-221, and gga-mir-1456, have been reported to be critical components in leukosis viral infection (Li et al., 2012). These results indicate that miRNAs may be important in virus infection or host immune responses.

Studies have revealed that mir-10b plays a significant role in breast cancer invasion and metastasis because it targets HOXD10, UPAR, and RHOC genes (Ma et al., 2007; Liu et al., 2012). Furthermore, it is also an important regulator of other kinds of cancer metastasis, such as malignant glioma and gastric cancer (Ma et al., 2010). It was highly expressed in the H9N2-infected fibroblast and differently expressed in the infected and non-infected groups in this study, which indicates that it could be an important regulator of viral infection. Additionally, it has been reported that mir-10a regulates the pro-inflammatory phenotype in atherosusceptible endothelium because it may inhibit HOXA1, MCP-1, IL-6, IL-8, and VCAM- 1 expression (Fang et al., 2010). mir-10a is also highly expressed in naturally occurring regulatory $\mathrm{T}$ cells, and regulates $\mathrm{T}$ cell differentiation by targeting BCL6 and NCOR2. Further studies are required to clarify the roles of gga-mir-10b, $-10 \mathrm{a}$, and $-181 \mathrm{a}$ in AIV infection.

gga- novel-1-mature may be a novel chicken miRNA or a virus miRNA. Not only was it highly expressed in the infected fibroblast, but it was also expressed differently between the infected and non-infected fibroblasts. Further studies are needed to better determine its prevalence and functions.

The results do not mean that all the differently expressed miRNAs are important in 
the immune response to viral infection. The most important function of some miRNAs may be in cell proliferation, apoptosis, other cell processes, etc. For example, gga-mir-126, ggamir-1456, and gga-mir-10b are differently expressed in the male and female gonads of chicken embryos. The results of pathway enrichment analysis show that miRNAs play an important role in regulating the cell cycle, such as ribosome biogenesis in eukaryotes, RNA transport, and arginine and proline metabolism, as well as the NF-kappa B signaling pathway, which is important for immune organ development, B cell maturation, and humoral immunity. Further studies are required to better understand the prevalence and functions of miRNAs.

\section{Conflicts of interest}

The authors declare no conflict of interest.

\section{ACKNOWLEDGMENTS}

We thank Dr. Quanjiao Chen for providing the AIV H9N2. Research supported by the Postdoctoral Project of Wuhan Academy of Agriculture Science and Technology, and partially supported by the Foundation of Hubei Postdoctoral Innovation Position and Wuhan Innovation and Talent Development funds.

\section{Supplementary material}

\section{REFERENCES}

Audic S and Claverie JM (1997). The significance of digital gene expression profiles. Genome Res. 7: 986-995.

Barber MR, Aldridge JR Jr, Fleming-Canepa X, Wang YD, et al. (2013). Identification of avian RIG-I responsive genes during influenza infection. Mol. Immunol. 54: 89-97.

Burnside J and Morgan R (2011). Emerging roles of chicken and viral microRNAs in avian disease. BMC Proc. 5: S2.

Chan LY, Kwok HH, Chan RW, Peiris MJ, et al. (2011). Dual functions of ginsenosides in protecting human endothelial cells against influenza H9N2-induced inflammation and apoptosis. J. Ethnopharmacol. 137: 1542-1546.

Clark MJ, Chen R, Lam HY, Karczewski KJ, et al. (2011). Performance comparison of exome DNA sequencing technologies. Nat. Biotechnol. 29: 908-914.

Cong YL, Pu J, Liu QF, Wang S, et al. (2007). Antigenic and genetic characterization of H9N2 swine influenza viruses in China. J. Gen. Virol. 88: 2035-2041.

Fang Y, Shi C, Manduchi E, Civelek M, et al. (2010). MicroRNA-10a regulation of proinflammatory phenotype in atherosusceptible endothelium in vivo and in vitro. Proc. Natl. Acad. Sci. U. S. A. 107: 13450-13455.

Guan Y, Shortridge KF, Krauss S and Webster RG (1999). Molecular characterization of H9N2 influenza viruses: were they the donors of the "internal" genes of H5N1 viruses in Hong Kong? Proc. Natl. Acad. Sci. U. S. A. 96: 9363-9367.

Guo Y, Li J and Cheng X (1999). Discovery of men infected by avian influenza A (H9N2) virus. Zhonghua Shi Yan He Lin Chuang Bing Du Xue Za Zhi 13: 105-108.

Hicks JA, Tembhurne PA and Liu HC (2009). Identification of microRNA in the developing chick immune organs. Immunogenetics 61: 231-240.

Lam WY, Yeung AC, Ngai KL, Li MS, et al. (2013). Effect of avian influenza A H5N1 infection on the expression of microRNA-141 in human respiratory epithelial cells. BMC Microbiol. 13: 104.

Langmead B and Salzberg SL (2012). Fast gapped-read alignment with Bowtie 2. Nat. Methods 9: 357-359.

Li QJ, Chau J, Ebert PJ, Sylvester G, et al. (2007). miR-181a is an intrinsic modulator of T cell sensitivity and selection. Cell 129: 147-61.

Li H, Ji J, Xie Q, Shang H, et al. (2012). Aberrant expression of liver microRNA in chickens infected with subgroup J avian leukosis virus. Virus Res. 169: 268-271.

Lian L, Qu L, Chen Y, Lamont SJ, et al. (2012). A systematic analysis of miRNA transcriptome in Marek's disease virusinduced lymphoma reveals novel and differentially expressed miRNAs. PLoS One 7: e51003. 
Liu Z, Zhu J, Cao H, Ren H, et al. (2012). miR-10b promotes cell invasion through RhoC-AKT signaling pathway by targeting HOXD10 in gastric cancer. Int. J. Oncol. 40: 1553-1560.

Liu D, Shi W, Shi Y, Wang D, et al. (2013). Origin and diversity of novel avian influenza A H7N9 viruses causing human infection: phylogenetic, structural, and coalescent analyses. Lancet 381: 1926-1932.

Ma L, Teruya-Feldstein J and Weinberg RA (2007). Tumour invasion and metastasis initiated by microRNA-10b in breast cancer. Nature 449: 682-688.

Ma L, Reinhardt F, Pan E, Soutschek J, et al. (2010). Therapeutic silencing of miR-10b inhibits metastasis in a mouse mammary tumor model. Nat. Biotechnol. 28: 341-347.

Meunier J, Lemoine F, Soumillon M, Liechti A, et al. (2013). Birth and expression evolution of mammalian microRNA genes. Genome Res. 23: 34-45.

Naeem K, Naurin M, Rashid S and Bano S (2003). Seroprevalence of avian influenza virus and its relationship with increased mortality and decreased egg production. Avian Pathol. 32: 285-289.

Peiris M, Yuen KY, Leung CW, Chan KH, et al. (1999). Human infection with influenza H9N2. Lancet 354: 916-917.

Powder KE, Ku YC, Brugmann SA, Veile RA, et al. (2012). A cross-species analysis of microRNAs in the developing avian face. PLoS One 7: e35111.

Schat KA and Purchase HG (1998). Cell culture methods. In: A Laboratory Manual for the Isolation and Identification of Avian Pathogens. 4th edn. (Swayne DE, Glisson JR, Jackwood MW, Pearson JE and Reed VWM, eds.). American Association of Avian Pathologists, Kennett Square, 223-234.

Scholtissek C and Rott R (1969). Ribonucleic acid nucleotidyl transferase induced in chick fibroblasts after infection with an influenza virus. J. Gen. Virol. 4: 125-137.

Terrier O, Textoris J, Carron C, Marcel V, et al. (2013). Host microRNA molecular signatures associated with human H1N1 and H3N2 influenza A viruses reveal an unanticipated antiviral activity for miR-146a. J. Gen. Virol. 94: 985995.

Wan H, Sorrell EM, Song H, Hossain MJ, et al. (2008). Replication and transmission of H9N2 influenza viruses in ferrets: evaluation of pandemic potential. PLoS One 3: e2923.

Wang Y, Brahmakshatriya V, Zhu H, Lupiani B, et al. (2009). Identification of differentially expressed miRNAs in chicken lung and trachea with avian influenza virus infection by a deep sequencing approach. BMC Genomics 10: 512.

Wang Y, Brahmakshatriya V, Lupiani B, Reddy SM, et al. (2012). Integrated analysis of microRNA expression and mRNA transcriptome in lungs of avian influenza virus infected broilers. BMC Genomics 13: 278.

Xu H, Yao Y, Smith LP and Nair V (2010). MicroRNA-26a-mediated regulation of interleukin-2 expression in transformed avian lymphocyte lines. Cancer Cell Int. 10: 15.

Yao Y, Zhao Y, Xu H, Smith LP, et al. (2008). MicroRNA profile of Marek's disease virus-transformed T-cell line MSB1: predominance of virus-encoded microRNAs. J. Virol. 82: 4007-4015.

Yao Y, Zhao Y, Smith LP, Lawrie CH, et al. (2009). Differential expression of microRNAs in Marek's disease virustransformed T-lymphoma cell lines. J. Gen. Virol. 90: 1551-1559.

Yao Y, Charlesworth J, Nair V and Watson M (2013). MicroRNA expression profiles in avian haemopoietic cells. Front. Genet. 4: 153.

Zhang H, Xu B, Chen Q and Chen Z (2011). Characterization of H9N2 influenza viruses isolated from Dongting Lake wetland in 2007. Arch. Virol. 156: 95-105. 